\title{
Fitobezoar Associado à Endometriose Intestinal: Uma Rara Causa de Obstrução Intestinal
}

\section{Intestinal Endemetriosis Associated to Phytobezoar: A Rare of Bowel Obstruction}

\author{
CARLOS ROBERTO AMORIM ${ }^{1}$; DIOGO MELGAÇO FARIA²; DILMAR DE CASTRO FILHO 3 ; \\ ROGÉRIO MENDES GRANDE'; VIRGÍNIOCÂNDIDOTOSTADESOUZA ${ }^{5}$
}

\begin{abstract}
${ }^{1}$ Professor auxiliar da Disciplina de Coloproctologia da Faculdade de Medicina da UNIVÁS (Universidade do Vale do Sapucaí - Pouso Alegre, MG). ${ }^{2}$ Acadêmico do quinto ano de medicina da UNIVÁS. ${ }^{3}$ Residente de Coloproctologia do Hospital das Clínicas Samuel Libanio. ${ }^{4}$ Médico Patologista do Hospital das Clínicas Samuel Libanio. ${ }^{5}$ Professor Titular do Departamento de Clínica Cirúrgica da UNIVÁS.
\end{abstract}

\begin{abstract}
AMORIM CR; FARIA DM; CASTRO FILHO D; GRANDE RM; SOUZA VCT. Fitobezoar Associado à Endometriose Intestinal: Uma Rara Causa de Obstrução Intestinal. Rev bras Coloproct, 2009;29(4): 501-504.

RESUMO: A endometriose intestinal é uma patologia benigna que afeta preferencialmente a porção retossigmóidea, sendo pouco frequente a localização em íleo terminal. Trata-se de um caso de uma paciente de 29 anos que foi admitida com dor abdominal difusa, náuseas e vômitos. Na propedêutica da obstrução intestinal a tomografia computadorizada revelou massa heterogênea em topografia ileocecal. Submetida a laparotomia exploradora onde foi feita a remoção de fibras vegetais mal digeridas aglomeradas em íleo terminal e ressecção de segmento íleoceco cólico devido a presença de estenose na válvula ileocecal. O exame anátomo patológico revelou endometriose intestinal e fitobezoar. A paciente evoluiu bem com alta no terceiro dia de pós operatório.
\end{abstract}

Descritores: Obstrução intestinal, Endometriose, Bezoares, Tratamento.

\section{INTRODUÇÃO}

A endometriose é uma desordem ginecológica frequente, crônica, benigna, dependente de estrógenos, associado à infertilidade e dor pélvica. Caracteriza-se pela presença de tecido endometrial em localização extra-uterina, ocorrendo com maior frequência no peritônio pélvico, ovários, septo retovaginal e intestino, sendo que este último caracteriza a endometriose intestinal. A presença de endometriose no íleo terminal é uma causa pouco freqüente de obstrução intestinal, que ocorre em $7 \%$ a $23 \%$ de todos os casos com comprometimento intestinal. ${ }^{(1,2)} \mathrm{A}$ incidência de ressecções intestinais devido à obstrução de intestino delgado por endometriose é de $0,7 \%$ dentre as pacientes operadas em decorrência de endometriose abdominopélvica. ${ }^{(2)}$

Como o próprio nome indica, fitobezoar consiste em material fibroso originado de vegetais e fru- tas, geralmente frutas cítricas e sementes. ${ }^{(3,4)}$ Estes elementos se acumulam originando um conglomerado de material não digerido e podem levar à obstrução intestinal, que representa uma grande complicação destas formações. ${ }^{(3)}$

A obstrução intestinal representa um quadro cuja etiologia pode ser extremamente variável. Dentre suas causas figuram desde patologias frequentes, como aderências pós-operatórias e hérnias, a mecanismos etiopatogênicos pouco habituais. Os autores apresentam um caso de obstrução intestinal secundária à coexistência de duas destas raras entidades clínicas: endometriose intestinal e fitobezoar.

\section{RELATO DO CASO}

R.A.P.T., 29 anos, feminino, leucoderma, natural e residente de Pouso Alegre-MG. Procurou o pron- 
to atendimento do Hospital das Clínicas Samuel Libânio na cidade de Pouso Alegre-MG relatando dor abdominal difusa do tipo colicas, de forte intensidade com início há quatro dias, associada a náuseas e vômitos. A paciente relata uso de Omeprazol devido à dores abdominais constantes.

Ao exame físico a paciente apresentava-se em bom estado geral, afebril, acianótica, anictérica, eupnéica e levemente desidratada. Os sinais vitais apresentavam-se normais. $\mathrm{O}$ exame abdominal revelou distensão moderada, doloroso à palpação de região epigástrica e presença de ruídos hidroaéreos à ausculta. O exame proctológico com retossigmoidoscopia rígida mostrou-se normal. Os exames laboratoriais revelaram leucocitose moderada e eletrólitos normais. $\mathrm{O}$ raio $\mathrm{X}$ de abdome e tórax mostrava distensão de alças de intestino delgado com níveis hidroaéreos e uma imagem radiopaca ovalada de aproximadamente $6 \mathrm{~cm}$ a direita. (Figura 1)

Optou-se pela internação da paciente, com jejum e hidratação venosa. Manteve o quadro de obstrução intestinal, com piora progressiva da distensão abdominal. Realizou-se TC que revelou massa heterogênea em topografia de válvula ileocecal, medindo $5,3 \mathrm{cmx}$ $2,5 \mathrm{~cm}$, presença difusa de níveis hidroaéreos e acentuada distensão de alças intestinais.(Figura 2)

Indicou-se laparotomia exploradora, onde foi observada distensão de alças de intestino delgado, obstrução de válvula ileocecal por corpo estranho. Foi realizada remoção de fitobezoar intraluminal que era composto de fibras de cítricos, fibras de manga e muitas sementes de mamão (Figura 3 e 4). Após minucioso estudo das alças intestinais observou-se uma estenose em válvula ileocecal, optou-se por colectomia de segmento íleocecal com anastomose íleo-ascendente termino-terminal (Figura 5).

A paciente evoluiu de forma satisfatória no pósoperatório e recebeu alta hospitalar três dias após a cirurgia. O exame anátomopatológico do segmento ressecado revelou tecido endometrióide em submucosa e túnica muscular de íleo terminal e válvula ileocecal (Figura 6), estabelecendo, portanto o diagnóstico de endometriose intestinal.

\section{DISCUSSÃO}

O intestino é envolvido em aproximadamente $10 \%$ dos casos de endometriose sendo que a porção mais comumente afetada é o retossigmóide, o que ocor-

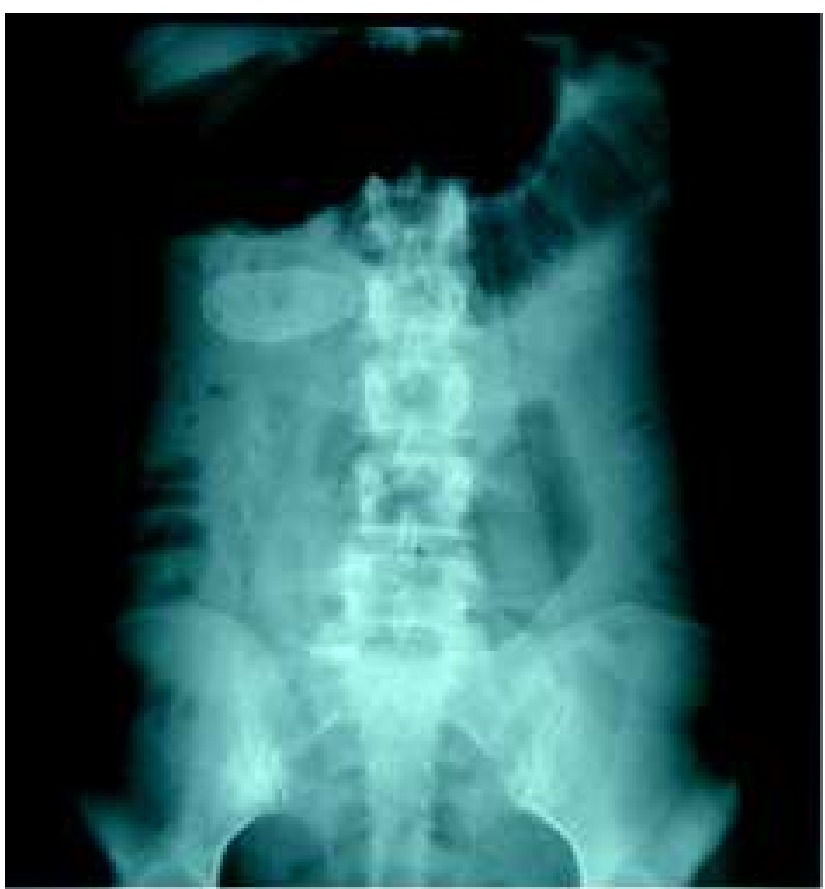

Figura 1 - Fitobezoar ao RX abdome.

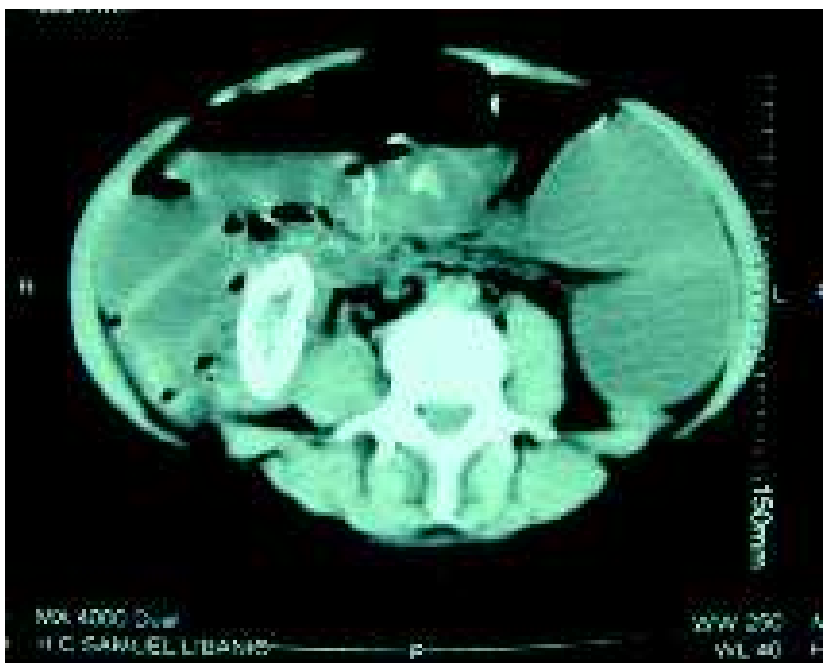

Figura 2 - TC abdome revelando massa em região ileocecal.

re em $90 \%$ dos casos. Outros segmentos afetados mais raramente são: o apêndice, íleo e ceco em 7\% dos casos, e intestino delgado em $3 \%$ dos casos. ${ }^{(2,5)}$

Os sintomas da endometriose intestinal podem ser em decorrência da lesão localizada ou de suas repercussões na fisiologia intestinal, quando há envolvimento da parede intestinal e consequente lesão dos plexos nervosos de Auerbach e Meissner. ${ }^{(6)}$ As queixas clínicas relatadas são: dor na região pélvica, dispareunia, alterações do hábito intestinal, sangramentos e dor durante evacuações, particularmen- 


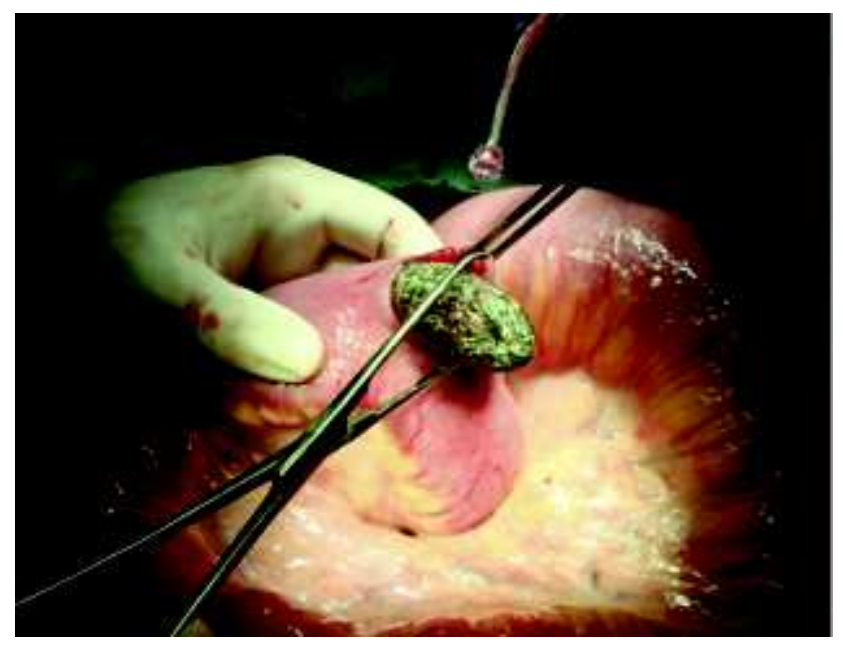

Figura 3 - Ileotomia com remoção de fitobezoar.

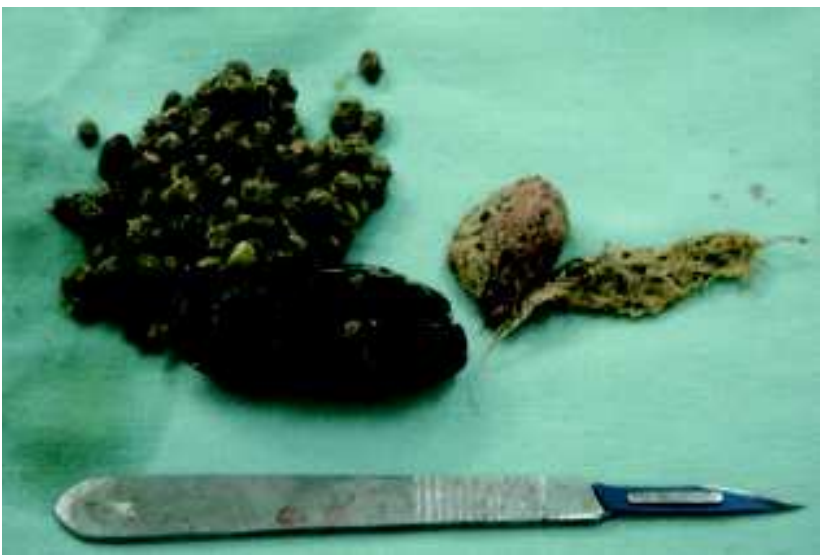

Figura 4 - Aglomerado de fibras vegetais e sementes-Fitobezoar.

te durante a menstruação. ${ }^{(2,5)}$ Sintomas como alterações da motilidade e do hábito intestinal assim como distensão abdominal também são relatados e podem levar ao diagnóstico errôneo de Síndrome do Intestino Irritável. ${ }^{(6)}$ Os sintomas se associam aos ciclos menstruais em aproximadamente $50 \%$ dos casos. ${ }^{(1,2)}$ Esta ciclicidade não foi estabelecida nesta paciente.

Dentre os quatro tipos de bezoar (fitobezoar, tricobezoar, lactobezoar e bezoares de remédios ou bolus de comida) os formados por fibras vegetais não digeridas são os mais comuns. A maioria dos pacientes que desenvolve fitobezoares possuem alguma alteração na capacidade digestiva ou motilidade gástrica( gastrectomia prévia,vagotomia, gastroparesia diabética ou distrofia muscular). ${ }^{(3,7)}$ Nenhuma destas alterações eram presentes na paciente do caso relatado.

A maioria dos pacientes portadores de bezoar relata vago desconforto epigástrico $(80 \%)$. Estes podem relatar também plenitude, náuseas ,vômitos,

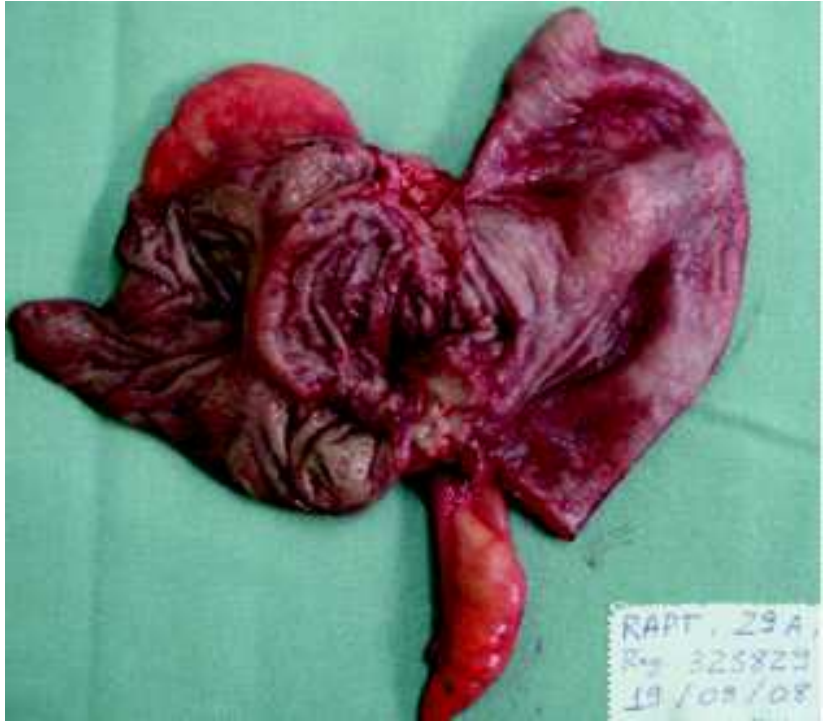

Figura 5 - Segmento ileocecal estenosado por endometriose intestinal.

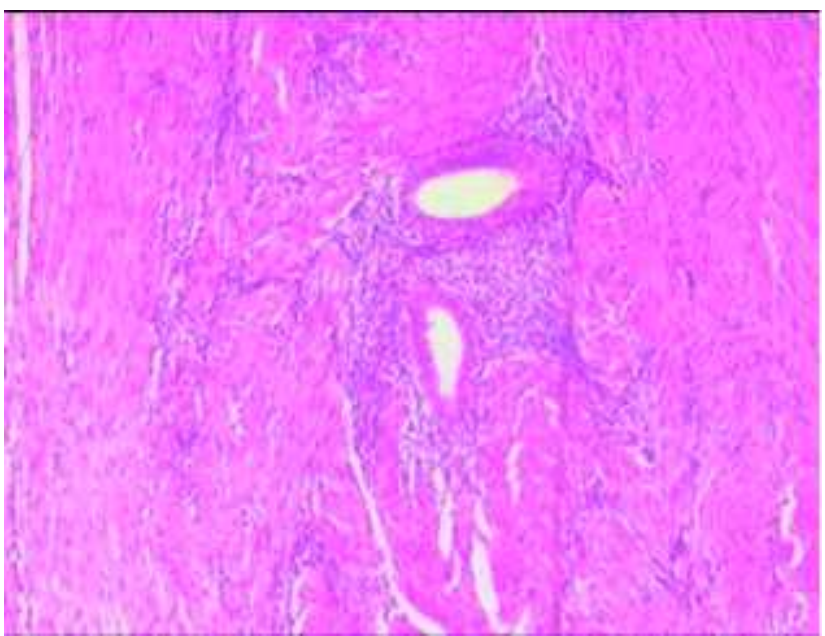

Figura 6 - Tecido endometrióide em túnica muscular de íleo terminal-coloração HE 40x.

disfagia e hemorragia digestiva alta. ${ }^{(3,8)} \mathrm{Um}$ artigo refere a ocorrência de saciedade precoce e perda de peso em $30 \%$ de pacientes portadores de bezoar. ${ }^{(3)}$

Um estudo feito com 14 pacientes cujo diagnóstico foi obstrução intestinal secundária a fitobezoar teve como distribuição dos mesmos: 2 pacientes $(14,3 \%)$ com obstrução de estômago, 5 casos de jejuno $(42,8 \%)$, em 6 pacientes a obstrução ocorreu em nível de íleo distal $(42,8 \%)$ e 1 apresentava fitobezoar em estômago e íleo $(7,1 \%)$. Destaca-se neste mesmo artigo uma correlação já bem estabelecida na literatura: aproximadamente $80 \%$ dos pacientes que apresentaram obstrução gastrointestinal por fitobezoar já ha- 
viam sido submetidos à cirurgia de redução da secreção ácida gástrica. ${ }^{(4)}$

Visto que a paciente deste relato não havia sido submetida a cirurgias prévias e não era portadora de patologias que predispõem ao desenvolvimento de fitobezoares, os autores supõem que a formação deste foi secundária á estenose do íleo terminal devido à infiltração do mesmo pela endometriose. No entanto, cabe destacar o uso de altas doses de medicação inibidora de bomba protônica (IBP) Omeprazol $40 \mathrm{mg}$ 2x/dia, e questionar se esta inibição da secreção ácida teria influenciado na digestão inadequada das fibras vegetais que vieram a se acumular originando o fitobezoar.

Quanto ao tratamento das patologias destacadas no artigo vale ressaltar a necessidade de cuidadosa busca de outros fitobezoares residuais ao longo do trato digestivo. ${ }^{(3,4,7)}$ No caso da endometriose, quando esta acomete toda a parede intestinal, a conduta é a ressecção do segmento afetado visto que a simples excisão do foco endometrióide não é curativa. ${ }^{(2,6)}$

As duas entidades clínicas citadas no presente trabalho são de difícil grau de suspeição no cenário da obstrução intestinal. Logo, são poucos os diagnósticos feitos no pré-operatório, sendo a maioria dos mesmos realizados durante $\mathrm{o}$ ato cirúrgico. ${ }^{(2,3)}$

Como conclusão cabe-se salientar a necessidade de, diante de um abdome agudo obstrutivo, sempre ter em mente as causas comuns de sua ocorrência mas também orientar a propedêutica e o diagnóstico diferencial para causas incomuns sejam de forma isolada ou até mesmo em associação com outras patologias, como o ocorrido no caso descrito pelos autores.

ABSTRACT: Intestinal endometriosis is a benign pathology, it occurs most often on the rectosigmoid portion, and the finding isn't very common on the terminal ileum. On this article the autors present a report from a 29 years old pacient, who had a difuse abdominal pain, nausea and vomiting. Computadorized tomography (CT) showed a heterogeneous mass on the ileocaecal area . An exploratory laparotomy was performed, badly metabolized vegetable fibers were removed from the terminal ileum, and a surgical removal of the ileocaecal -colic portion was done, since there was a stenosis on that area. The anatomical-pathologic study showed intestinal endometriosis and phytobezoar The patient had a good follow up and got out the hospital on the third day after surgery.

Key words: Intestinal obstruction. Endometriosis, Bezoars, Treatment.

\section{REFERÊNCIAS}

1. Beltrán MA, Tapia F, Araos F, Martinéz H, Cruces KS. Ileal endometriosis as a cause of intestinal obstruction. Report of two cases. Rev Méd Chile 2006; 134: 485-490

2. De Ceglie A, Bilardi C, Blanchi S, Picasso M, Di Muzio M, Trimarchi A, Conio M. Acute small bowel obstruction caused by endometriosis: A case report and review of the literature. World J Gastroenterol 2008; 14(21) : 3430-3434

3. Andrus $\mathrm{CH}$, Ponsky JL. Bezoars: classification, pathophysiology, and treatment. Am J Gastroenterol. 1988; 83(5):476-8

4. Rubin M, Shimonov M, Grief F, Rotestein Z, Lelcuk S. Phytobezoar: a rare cause of intestinal obstruction. Dig Surg. 1998; 15(1): 52-4

5. Podgaec S, Gonçalves MO, Klajner S, Abrão MS. Epigastric pain relating to menses can be a symptom of bowel endometriosis. Sao Paulo Med. J. vol.126 no.4 São Paulo July 2008
6. Preziosi G, Cristaldi M, Angelini L. Intestinal obstruction secondary to endometriosis: A rare case of synchronous bowel localization . Surg Oncol. 2007; 16, Suppl 1: S161-3

7. Schoeffl V, Varatorn R, Blinnikov O, Vidamaly V. Intestinal obstruction due to phytobezoars of banana seeds: a case report. Asian J Surg;27(4):348-51, 2004 Oct.

8. Pitiakoudis M; Koukourakis M; Giatromanolaki A; Tsaroucha AK; Polychronidis A; Simopoulos C. Phytobezoars as a cause of small bowel obstruction associated with a carcinoid tumor of the ileocecal area. Acta Chir Iugosl;50(2):131-3, 2003.

Endereço para correspondência:

CARLOS ROBERTO AMORIM

Rua Benedita Alves Funchal, 81 Apto 304

Bairro Jd Guanabara,

Pouso Alegre, MG

CEP 37.550.000

Tel.: (35) 9984-6091 / (35) 3421-9380

E-mail: amorimcr@yahoo.com.br 